\title{
Future Treatment of Sleep Disorders
}

\section{Syndromic Approach Versus Management of Treatable Traits?}

\author{
Dirk Pevernagie, $\mathrm{MD}, \mathrm{PhD}^{\mathrm{a}, \mathrm{b}, *}$
}

\section{KEYWORDS}

- Sleep disorders • Sleep medicine $\bullet$ Obstructive sleep apnea $\bullet$ Endotype $\bullet$ Phenotype

- Precision medicine $\bullet$ Treatable trait $\bullet$ Fallacy

\section{KEY POINTS}

- Sleep medicine is cataloged according to a conventional disease classification system. Disease models are rooted in the pathophysiology of sleep. Polysomnography and other tests are used to demonstrate pathophysiological mechanisms underlying the currently known sleep disorders.

- Although many patients with sleep disorders may be adequately managed by this pathophysiological approach, therapeutic results are insufficient in some subjects, the causes of which lie in nonspecificity of symptoms, coincidental association between symptoms and pathophysiological endotype, as well as co-occurrence of two or more pathologic mechanisms affecting sleep.

- As co-occurrence of different pathogenetic mechanisms may produce phenotypes that are at odds with the idealized description of classic sleep disorders, the result of standard therapeutic interventions may be disappointing.

- The mechanisms underlying the expression of certain traits may be a substrate for targeted treatment. Treatable traits are characterized by biomarkers with predictive value as to beneficial treatment response.

- The challenge for the future is to gradually embrace the principles of systems medicine and to shift gear toward managing treatable traits in sleep disorders surpassing the limits of the traditional nosologic approach.

\section{INTRODUCTION}

Over the past decades, sleep medicine has evolved as a novel discipline in health care. The development of relevant medical specialties has invariably been preceded by major scientific advances in particular areas of interest. Medical and surgical specialties have traditionally been organized on anatomic or organ-based models in line with growing insight in organ-system physiology and pathology. The taxonomy of human disease dates back to the nineteenth century and is largely ascribed to the work of Sir William Osler, one of the founding fathers of modern medicine. ${ }^{1}$ The classification of diseases by connecting the affected organ system with physiologic, anatomic, and histologic findings has been called the "Oslerian paradigm." ${ }^{2}$ Syndromic patterns and nosologic entities are the building blocks of the Oslerian taxonomy that still prevails in the contemporary classification of human diseases.

Later in medical history, cross-sectional disciplines have emerged that are rooted in common

\footnotetext{
a Department of Respiratory Medicine, Ghent University Hospital, Corneel Heymanslaan 10, 9000 Gent, Belgium; b Department of Internal Medicine and Paediatrics, Faculty of Medicine and Health Sciences, Ghent University, Corneel Heymanslaan 10, 9000 Gent, Belgium

* Corresponding author.

E-mail address: dirk.pevernagie@ugent.be
} 
biological settings and that integrate different organ systems in a particular context. Relevant "horizontal" disciplines have been developed in age domains (pediatrics and geriatrics), cell biology (oncology), microbiology (infectiology), to name a few. Sleep is an essential biological process that can be readily impaired by pathophysiological mechanisms. Evidently, the various sleep disorders have a common ground underpinning the concept of clinical sleep medicine as we know it today. The technological revolution over the past century has instigated sleep research, thereby disclosing a vast amount of scientific information and producing exquisite tools for diagnosing and treating sleep disorders. This evolution has paved the way for setting up sleep medicine as a medical discipline in its own right. ${ }^{3}$ In line with this development, curricula in sleep medicine have been established uplifting it on a par with educational standards in other disciplines. ${ }^{4}$

In parallel with the creation of a professional title, textbooks, guidelines, and catalogs for disease classification have been published. The International Classification of Sleep Disorders (ICSD), issued by the American Academy of Sleep Medicine (AASM), is a concise reference book that systematically classifies the currently known disease entities of sleep. ${ }^{5}$ In this manual, sleep disorders are categorized into various domains, including insomnia, sleep-disordered breathing, central hypersomnia, circadian rhythm disorders, parasomnia, sleep-related movement disorders, and miscellaneous conditions. The sleep disorders themselves are described by essential and associated features, predisposing and precipitating factors, natural course, pathophysiology, as well as results from polysomnography (PSG) and other objective tests. Basically, the items listed in the nosologic classification of the ICSD are modeled as disease entities. The disease model consists of a constellation of symptoms and signs complemented with characteristic pathophysiological findings on PSG (and other complementary tests). The merger of clinical findings and observations from diagnostic testing is deemed specific with respect to causality. The connotation of causality is reinforced by adding the term "syndrome" to certain disorders, for example, "sleep apnea syndrome" and "restless legs syndrome." Moreover, the ICSD provides diagnostic criteria for each nosologic entity. These criteria commonly include a mixture of symptoms, signs, and objective PSG findings. Diagnostic cutoffs are typically based on frequency and/or severity ratings of symptoms and PSG characteristics.

Inherently, a cause-consequence relationship is inferred for each sleep disorder listed in the ICSD, the cause being a pathophysiological process demonstrable by PSG or other methods and the consequence being the clinical presentation. However, symptoms and signs overlap between nosologic entities and often correlate poorly with the degree of pathophysiological abnormality assessed by objective tests. ${ }^{6}$ Not infrequently, the recommended therapy for sleep disorders fails to produce symptomatic relief or is not well tolerated, suggesting that the cause is not really affected by the treatment. In these cases, there are reasons to believe that causality is uncertain and that apparent or concealed confounders influence or determine the outcome. Thus, the question arises whether sleep medicine practice of the future should stick to the conventional "syndromic" approach or rather move to management of clinical traits that are likely to respond to targeted treatment?

\section{Nosological Classification of Sleep Disorders: Evolution and Intrinsic Weaknesses}

The AASM has revised the ICSD on several occasions. The nosologic classification of sleep disorders has been adjusted to integrate new scientific data. More emphasis has been placed on the role of pathophysiological observations on PSG. As a consequence, the theoretic concept of certain sleep disorders has evolved. To illustrate some conceptual adaptations over time, the present discussion will focus on the evolution of obstructive sleep apnea (OSA) as a model of chronic sleep disturbance across the consecutive editions of the ICSD, also highlighting some logical errors that inadvertently have crept in.

The first edition of the ICSD was published in 1990 by the American Sleep Disorders Association (the predecessor of the AASM). ${ }^{7}$ The diagnostic criteria for OSA syndrome did not include any count of respiratory events nor the apneahypopnea index (AHI), but only a qualitative description: "frequent episodes of obstructed breathing." The severity criterion was primarily based on the seriousness of symptoms, which was assumed would be reflected in the PSG findings.

In 2005, The AASM published the second edition of the ICSD (ICSD-2). ${ }^{8}$ The new version was at odds with the previous one in that $\mathrm{AHI}$ cutoff points were presented as the primary criterion for the definition of OSA. A diagnosis of OSA could be established based on an $\mathrm{AHI}$ greater than or equal to $5 / \mathrm{h}$ in the presence of symptoms or an $\mathrm{AHI}$ greater than or equal to $15 / \mathrm{h}$ even without symptoms. The cutoff points were inspired by an earlier AASM publication on OSA syndrome 
definition and measurement techniques. ${ }^{9}$ In this paper, the AHI was introduced as a metric for gauging OSA severity. This proposition was based on a single cross-sectional population survey that showed an association between $\mathrm{AHI}$ and prevalent hypertension..$^{10}$ However, because this study did not include any prospective data at the time of publication, causal inference was scientifically inappropriate. Nevertheless, AHI was from then on accepted as the primary measure of OSA severity.

The AHI-driven remodeling of OSA has introduced a converse error (Box 1). ${ }^{11,12}$ This error of reasoning reverses the logical order of premise

\section{Box 1 \\ Logical relation between clinical presentation and increased apnea-hypopnea index in obstructive sleep apnea

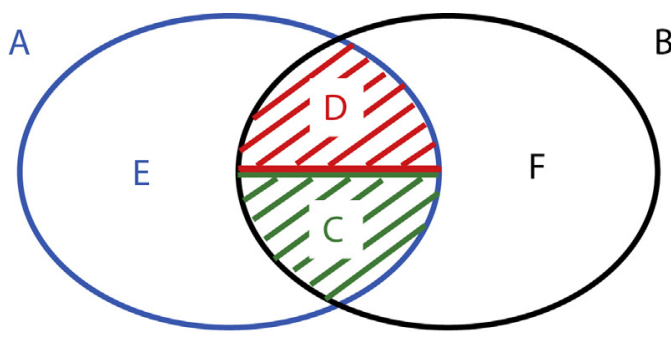 \\ A: symptoms and signs that suggest OSA \\ $B$ : increased AHI \\ $C$ : causally related $A$ and $B$ (true positive) \\ $D$ : coincidentally related $A$ and $B$ (false positive) \\ E: A with normal AHI \\ F: B without symptoms and signs that suggest OSA}

Using B as a predictor for clinically relevant, treatment responsive OSA brings about fallacies:

Set $B$ is a source of the McNamara or quantitative fallacy: $\mathrm{AHI}$ is a test result. Although it is commonly used as a metric of disease state, it misrepresents the clinical characteristics and severity of the disorder.

Set $F$ is congruent with division fallacy: assuming that an increased $\mathrm{AHI}$ invariably represents symptomatic disease is erroneous. Many subjects are asymptomatic.

Sets $C, D$, and $F$ are related to converse error (aka affirming the consequent): patients with true OSA (set C) have an increased AHI-the opposite is not necessarily true.

Set $D$ represents association fallacy: false diagnosis of true OSA based on a coincidental association between $A$ and $B$. and consequent: subjects with clinically relevant OSA have an increased $\mathrm{AHI}$, but the converse is not necessarily true. The relevance of this error has become evident in epidemiologic research. In a Swiss survey on middle-aged to older people in an urban community, it was shown that the prevalence of an $\mathrm{AHI}$ greater than or equal to $15 / \mathrm{h}$ amounted to $49.7 \%$ in men and $23.4 \%$ in women. ${ }^{13}$ In most of these people, daytime sleepiness or other symptoms of OSA were absent. Because an increased $\mathrm{AHI}$ can be demonstrated in large percentage of asymptomatic subjects in the general population, doubt must be casted on the validity of the $\mathrm{AHI}$ as meaningful metric of OSA disease severity. ${ }^{6}$ Hence it follows that converse error will continue to distort the OSA disease model as long as the AHI is maintained as the prime predictor variable.

Box 1 also illustrates other deficiencies in reasoning related to false associations and assumptions. ${ }^{11}$ In the ICSD-2, nosologic entities are described as sets of symptoms, signs, and PSG findings representing disease profiles with a common pathophysiological denominator. However, several sleep disorders have heterogeneous manifestations that do not simply fit a disease model cast into a concise set of diagnostic criteria. This reductionist approach may surely apply to certain subgroups, but by no means can it apply to all patients of the entire target group. Division fallacy is wrongly assuming that an individual belonging to a group (eg, subjects with an $\mathrm{AHI} \geq$ $15 /$ h) necessarily show other key characteristics of that group (eg, suffering from daytime sleepiness). It is often assumed that symptoms of OSA and increased $\mathrm{AHI}$ are causally related. Because this association can be due to coincidence, it is also a misconception. The quantitative (aka McNamara) fallacy is yet another misconception in which decisions rely solely on one metric, thereby ignoring all other observations. ${ }^{14}$ The presumption that $\mathrm{AHI}$ by itself represents a disease state of OSA in a dose-dependent manner is not justified. The correlation between $\mathrm{AHI}$ and clinical manifestations is weak at best. Ascribing metric properties to the $\mathrm{AHI}$ is obviously an overqualification. ${ }^{6}$

The third edition of the ICSD (ICSD-3) has further expanded on OSA as a disease model, including mental, metabolic, and cardiovascular comorbidities as intrinsic components of the disorder. ${ }^{15}$ Yet, no additional evidence was put forward in this edition to support the assumption that the $\mathrm{AHI}$ reflects clinical disease severity. Despite this omission, the ICSD-3 has been quoted as a reference for OSA severity rating in a recent clinical guideline on diagnostic testing of OSA published by the AASM. ${ }^{16}$ The ICSD-3 takes the disease model even one step further, deleting the ultimate 
criterion described in IDSC-2 that "the disorder [ie OSA] is not better explained by another current sleep disorder, medical or neurologic disorder, medication use, or substance use disorder." The ICSD-3 clearly excludes the need for differential diagnosis and, as such, endorses association fallacy.

The aforementioned fallacies may have farreaching consequences for clinical research and daily practice. There is as yet no gold standard (or "ground truth") to define the real disease state of OSA. The causative role of OSA in provoking symptoms and signs is hard to ascertain, even in the presence of high $\mathrm{AHI}$ values. As mentioned earlier, a coincidental association between an indicative clinical picture and increased $\mathrm{AHI}$ may be labeled as "false positive" OSA. In clinical practice, false-positively labeled patients with OSA will experience little benefit of therapy and may show poor adherence. Trying to optimize compliance to therapy in these individuals will not improve the clinical results. In clinical research, the outcomes of randomized controlled trials may be blurred by mixing up false-positive and truepositive OSA. The customary inclusion criterion of AHI greater than threshold will include both categories without knowing who's who. Obviously, the $\mathrm{AHI}$ bias will have to be addressed to improve future results in both clinical practice and research. ${ }^{17}$

\section{New Perspective: Studying Heterogeneity and Complexity of Sleep Disorders}

Chronic diseases are hallmarked by multicomponent and nonlinear pathologic processes and are ill-suited to be comprehended by reductionist models. ${ }^{18}$ Instead, systems science offers tools to effectively treat susceptible traits at an individual level. Alvar Agusti has pioneered such approach in asthma and chronic obstructive pulmonary disease (COPD), two highly prevalent and disabling chronic diseases. ${ }^{19}$ Taking obstructive lung disease as a starting point, he has elegantly described the historical transition of medical reasoning. Disease management, traditionally based on pathology- and pathophysiology-oriented diagnosis, had at some point to be fine-tuned. The first step was to identify differential disease attributes in patients with a common diagnosis allowing stratification into clinical phenotypes. However, such subclassification also proved insufficient to predict therapeutic effects and prognosis within the stratified subgroups. Eventually, it became obvious that a subsequent step had to be taken and that assessment of disease characteristics was required at the individual level. ${ }^{20}$

At present, personalized (aka "precision") medicine is proposed as the ultimate paradigm to overcome the limitations of the former strategies. It is defined as "treatments targeted to the needs of individual patients on the basis of genetic, biomarker, phenotypic or psychosocial characteristics that distinguish a given patient from other patients with similar clinical presentations." ${ }^{21}$ The main objective of precision medicine is to "improve clinical outcomes for individual patients while minimizing unnecessary side effects for those less likely to respond to a given treatment." 22 The rationale for assuming personalized medicine is the observation that chronic diseases are "complex" and "heterogeneous." In this setting, "complex" means that they have several components with nonlinear dynamic interactions, whereas "heterogeneous" indicates that not all of these components are present in all patients or, in a given patient, at all timepoints. ${ }^{21}$ An explanation of these concepts is presented in Table 1.

The transition to personalized medicine is rooted in systems biology. ${ }^{23}$ This scientific domain studies the complex time, space, and contextsensitive interactions of the vast amount of components that constitute a biological system. Information is lost by zooming in on the individual components. In order to gain new insights, the dynamics of the entire system must be analyzed at an integrated meta-level. Analytical methods are derived from systems engineering and big data science. The transposition of systems biology to the scientific domain of medicine is called "systems medicine" or "network medicine." 24 The intricate interaction of processes at environmental, clinical, biological, and genetic levels ultimately defines the clinical outcomes. In brief, the study of these complex mechanisms is grouped into basic domains covering genotypes, endotypes, and phenotypes (Table 2). ${ }^{24}$ Meanwhile, the principles of precision medicine have been reviewed and deemed appropriate for common practice by opinion leaders of the sleep medicine community. ${ }^{25,26}$

\section{Precision in Sleep Medicine}

Endotypes and phenotypes have been studied intensively over the past decade, especially in the domain of sleep-disordered breathing. ${ }^{27}$ An endotype denotes a particular mechanism that causes a physiologic or metabolic disturbance in certain organ systems. In OSA, several physiologic endotypes have been observed that, together, shape the disturbed breathing process. Members 


\begin{tabular}{|c|c|c|c|}
\hline $\begin{array}{l}\text { Type of Disease } \\
\text { Management } \\
\end{array}$ & Underlying Concept & Clinical Implications & Management \\
\hline $\begin{array}{l}\text { Traditional } \\
\text { medicine }\end{array}$ & $\begin{array}{l}\text { Monodimensional, uniform } \\
\text { disease processes }\end{array}$ & $\begin{array}{l}\text { Syndromic approach: a } \\
\text { common denominator of } \\
\text { observed symptoms, } \\
\text { signs, and pathologic } \\
\text { markers defines the illness }\end{array}$ & "One size fits all" \\
\hline $\begin{array}{l}\text { Stratified } \\
\text { medicine } \\
\end{array}$ & $\begin{array}{l}\text { Heterogeneity within } \\
\text { nosologic entities }\end{array}$ & $\begin{array}{l}\text { Phenotyping, stratification } \\
\text { of subtypes }\end{array}$ & $\begin{array}{l}\text { "One size fits every } \\
\text { subtype" }\end{array}$ \\
\hline $\begin{array}{l}\text { Personalized } \\
\text { medicine }\end{array}$ & $\begin{array}{l}\text { Heterogeneity plus } \\
\text { complexity within } \\
\text { nosologic entities }\end{array}$ & $\begin{array}{l}\text { Multiple causes or } \\
\text { pathologic processes may } \\
\text { underly discrete } \\
\text { phenotypes. } \\
\text { Discrimination between } \\
\text { conventional nosologic } \\
\text { entities becomes less } \\
\text { obvious }\end{array}$ & $\begin{array}{l}\text { Label-free, targeted therapy } \\
\text { for treatment-responsive } \\
\text { traits }\end{array}$ \\
\hline
\end{tabular}

\begin{tabular}{|c|c|}
\hline Term & Meaning \\
\hline Exposome & $\begin{array}{l}\text { The cumulative/lifelong environmental exposures including } \\
\text { smoking, pollution, noxious substances, infections, and diet. }\end{array}$ \\
\hline Genome & $\begin{array}{l}\text { The total composition of genes in a cell defining the genetic make- } \\
\text { up of an organism or individual. }\end{array}$ \\
\hline Epigenetics & $\begin{array}{l}\text { Molecular mechanisms/multilevel biological networks that } \\
\text { dynamically modulate the outcome of gene-environment } \\
\text { interactions. }\end{array}$ \\
\hline Genotype & $\begin{array}{l}\text { The part of the genome (ie, a gene or set of genes) that codes for } \\
\text { the characteristics of an organism or individual, determining the } \\
\text { phenotype through the intermediary pathway of endotypes. }\end{array}$ \\
\hline Endotype & $\begin{array}{l}\text { The subtype of a condition that has a distinct molecular, functional, } \\
\text { or pathobiological mechanism. Studying endotypes allows } \\
\text { mechanistic approaches to disease stratification and treatment } \\
\text { beyond the clinical presentation of the disease. }\end{array}$ \\
\hline Phenotype & $\begin{array}{l}\text { Observable characteristics of an organism or individual in health } \\
\text { and disease. A combination of disease features, in relation to } \\
\text { clinically meaningful attributes (symptoms, response to therapy, } \\
\text { health outcomes, quality of life). }\end{array}$ \\
\hline Trait & $\begin{array}{l}\text { A particular characteristic such as an endotype or clinical subtype. A } \\
\text { treatable trait is a therapeutic target identified by recognition of } \\
\text { phenotype or endotype through validated biomarkers. }\end{array}$ \\
\hline Biomarker & $\begin{array}{l}\text { A measurable indicator (biological molecule in body fluids as well as } \\
\text { physiologic phenomenon) used to gauge a particular biological } \\
\text { or pathogenic process or response to treatment. Validated } \\
\text { biomarkers may be reliable surrogates for certain endotypes or } \\
\text { phenotypes. }\end{array}$ \\
\hline Cluster & $\begin{array}{l}\text { A set of characteristics that together point at a common cause or } \\
\text { pathogenic mechanism }\end{array}$ \\
\hline
\end{tabular}


from the Division of Sleep Medicine at the Brigham and Women's Hospital (Harvard Medical School) have explored the pathophysiological mechanisms of sleep-disordered breathing. ${ }^{28}$ Briefly, they demonstrated that several factors play a role in upper airway obstruction, including an anatomic feature predisposing to collapsibility of the upper airway, and nonanatomical traits (genioglossus muscle responsiveness, arousal threshold, and respiratory control stability-loop gain). In subsequent studies it was found that the nonanatomical pathophysiological traits are suitable to therapeutic options other than the conventional application of positive airway pressure therapy or the use of oral appliances. ${ }^{29}$ More specifically, supplemental oxygen therapy or carboanhydrase inhibitors may be effective at reducing loop gain, whereas hypnotics may increase the arousal threshold, and upper airway muscle training or hypoglossal nerve stimulation may compensate for insufficient genioglossus muscle responsiveness. ${ }^{27,30,31}$ Evidence is accumulating that physiologically targeted treatment of OSA may effectively decrease the $\mathrm{AHI}$ and thus lower the pathophysiological burden of the disorder. However, it is as yet uncertain whether this would also translate into reduced symptom scores.

Although the term "phenotype" can have different meanings, it usually denotes a combination of disease characteristics, in relation to clinically meaningful attributes (symptoms, treatment response, health outcomes, quality of life) that can be used to distinguish certain categories of patients from others. ${ }^{32,33}$ Within taxonomically defined diseases such as OSA and COPD, the observed clinical heterogeneity necessitates subclassification into different clinical phenotypes. Cluster analysis is a suitable statistical method to discern subtypes in large heterogeneous groups. This technique has been explained in a recent review by Zinchuk and colleagues. ${ }^{34}$ Cluster analysis uses hypothesis-generating (unsupervised) learning methods for discovering patterns in the parent population by grouping individuals into homogeneous categories, based on the clustering of particular features. The results depend on the parameters fed into the model and may focus on different outcomes including symptoms, PSG features, treatment results, and risk for having or developing comorbidities.

Cluster analysis has revealed remarkable findings in OSA research. Basically, three symptomatic forms of OSA have been identified, namely patients with disturbed sleep, minimal symptoms, and excessive sleepiness. ${ }^{35}$ These observations have been replicated and expanded in a subsequent international, multicentric study. ${ }^{36}$ In addition to the three basic clusters two other categories were proposed, that is, upper airway symptoms dominant and sleepiness dominant subtypes. Similar average $\mathrm{AHI}$ values were found in both study samples and across clusters, indicating that clinical phenotypes cannot be differentiated by the AHI. The disturbed sleep or "insomnia" phenotype was observed to be dominant in a survey of the European Sleep Apnoea Database. ${ }^{37}$ In this study, phenotypes with insomnia symptoms comprised more than half of the patients with OSA and were more frequently linked with cardiovascular comorbidity than those with excessive sleepiness, despite less severe OSA. Using data from the Sleep Heart Health Study, the excessively sleepy subtype was found to be most strongly associated with prevalent heart failure and incident cardiovascular disease. ${ }^{38}$ Cluster analysis of a multisite US Veteran cohort disclosed 7 OSA subtypes, when PSG data were fed into the statistical analysis. ${ }^{39}$ In this study, certain physiologic endotypes (including those with periodic limb movement of sleep) captured risk of adverse cardiovascular outcomes, which was missed by the conventional AHI-based OSA severity classification.

Obviously, disparate results from cluster analyses regarding clinical phenotypes in OSA may reflect differences in patient samples and methods used. As yet, insufficient data exist to confirm that phenotypic clustering may be reproducible within the same datasets or across cohorts. ${ }^{34}$ In accordance with other clinical trials, the target population for cluster analysis studies are subjects referred for OSA evaluation who happen to have an $\mathrm{AHI}$ greater than threshold. As previously explained, nonspecific symptoms such as insomnia or excessive sleepiness do not become specific markers of OSA in the presence of an increased $\mathrm{AHI}$, and association fallacy may render the outcome of cluster analysis trials noninterpretable. The AHI should be considered the catch-22 of clinical OSA research. Addressing the "AHI bias" will be a major challenge for future clinical research.

Finally, OSA is not only complex and heterogeneous but also overlaps and co-occurs with other sleep disorders. Moreover, lifestyle characteristics and manifestations of medical and psychiatric comorbidities may further blend in with the palette of symptoms, thereby generating composed phenotypes. In order to predict responses to therapeutic interventions more reliably, unique mechanistic traits and clinical features of OSA will have to be determined. ${ }^{27}$

\section{The Quest for Specificity: Discovering Markers that Indicate Treatable Traits}

From the earlier discussion it becomes evident that the top-down Oslerian approach to disease, 
starting with the clinical presentation of the disorder at the top, followed by identifying the pathophysiological mechanisms below, ${ }^{24}$ does not always work for sleep disorders medicine. In many patients with OSA the pathophysiological endotype does not seem to match the clinical phenotype. ${ }^{27}$ The one-size-fits-all therapeutic strategies fail in a sizable number of patients categorized according to the prevailing ICSD. Cluster analysis has produced variable results and no consistent actionable information has come forth as to guide specific therapy in identified phenotypes. Furthermore, hypothesis-driven phenotyping is prone to circular reasoning, predisposing subjects to being labeled with the diagnostic outcome already before the diagnostic procedure has started (eg, "subjects referred for OSA evaluation"). Therefore, there is an opportunity for sleep medicine to leave the beaten path of Oslerian classification and to gradually adopt the principles of precision medicine.

To find markers that enhance specificity in the relationship between pathophysiological endotypes and the clinical phenotypes is a challenge of high priority. As clinical phenotypes in sleep medicine are often characterized by a set of nonspecific symptoms and signs, the identification of treatable traits is of prime importance. Traits can be considered as identifiers of causality between a pathogenetic mechanism and its clinical expression in the context of a disease model. The connotation "treatable" means that a trait may qualify as a decision aid for administering a targeted treatment that is expected to be effective. Treatable traits are not necessarily mutually exclusive. They can coexist in the same patient and can change with time. ${ }^{24}$

In OSA, the primary token of specificity regarding the relation between endotype and phenotype is a favorable response to therapy. ${ }^{6}$ However, this is a post hoc finding of an ordinary trial-and-error approach, which is not quite appealing in the setting of modern medicine. The challenge is indeed to discover treatable traits that not only assure success of lowering the $\mathrm{AHI}$ but also, first and foremost, predict symptomatic response to targeted treatment. In recent years, investigators have sought to improve the diagnostic yield of clinical sleep studies. PSG offers exquisite multichannel and multivariable technology suited to demonstrate intricate pathophysiological mechanisms. It seems that routine PSG can be enhanced to emulate the (obtrusive and invasive) techniques that were used to validate the four endotypes described by Eckert and colleagues. ${ }^{28,40,41}$ Furthermore, intelligent digital algorithms can be applied to expand the array of
PSG metrics, such as sleep depth, arousal characteristics, and hypoxic burden, among others. ${ }^{42}$ The big data generated by thousands of polysomnographic recordings are an excellent substrate for novel analytical approaches. Innovative scoring techniques are expected to come out of the application of artificial intelligence. For example, hypnographic analysis of sleep has revealed a new sensitive and specific marker for type 1 narcolepsy, in the form of an unusual overlap between sleep stages. ${ }^{43}$ Thus, innovation of PSG methodology holds promise for new biomarkers with a potential to serve as treatable traits.

The presence of soluble biomarkers in body fluids or exhaled air is another exciting field of scientific discovery. The study by Sànchez-de-laTorre and colleagues was one of the first to show that biomarkers are capable of predicting treatment responses in patients susceptible to the hypertensive effects of OSA. ${ }^{44}$ In this study, the presence of a particular microRNA profile was associated with the likelihood that blood pressure would be reduced by CPAP therapy in OSA patients with resistant hypertension. It can be expected that such information may assist in future decision-making regarding administration of CPAP versus prescribing antihypertensive drugs in this target group. In the domain of epigenetics, exploration of the transcriptome holds promise for the discovery of new biomarkers. ${ }^{45}$ The noncoding RNAs, specifically microRNAs, seem relevant as potential indicators for the management of OSA, and the potential translational applicability of these molecules extends beyond predicting effects of CPAP on blood pressure. ${ }^{45}$ Other potential biomarkers for OSA have been searched in the domain of proteomics. ${ }^{46}$ Although some candidate markers have been identified, their accuracy is as yet insufficient to be implemented in clinical practice. Overall, the application of "-omics" seems promising for defining treatable traits, but the scientific development is still in its infancy. With the application of artificial intelligence and big data statistical analysis it is expected that useful biomarkers will emerge and will be integrated into clinical practice in the years to come.

In conclusion, it has become evident that the present nosologic classification of sleep disorders is too restricted. In the ICSD, sleep disorders have been parceled out into separate disease entities based on traditional taxonomy and pathophysiological modeling. In daily practice, however, nonspecificity of symptoms may confound diagnostic decision-making. Moreover, blended clinical conditions are frequently encountered for which clues as to choosing 
efficient and effective treatment are lacking. Therefore, it is time to downscale the Oslerian principle of disease management and to integrate systems medicine-based, unbiased ("label-free") approaches allowing effective treatment of traits that may be shared by different sleep disorders.

\section{CLINICS CARE POINTS}

- The treatment of sleep disorders is often straightforward, much to the satisfaction of the patient and the medical doctor. Patients presenting with complex phenotypes, however, may pose therapeutic problems.

- Treatment failure in clear-cut cases or problems in patients with complex phenomenology should prompt for other solutions than conventional therapy.

- The identification of traits that are responsive to targeted treatment is paramount in these cases.

- Although systems medicine-the driving force behind the development of personalized medicine-is at its infancy in the domain of sleep disorders, recent developments hold potential for diagnostic and therapeutic innovations that expectedly will become available for routine practice in the not-sodistant future.

\section{DISCLOSURE}

The author has nothing to disclose.

\section{ACKNOWLEDGMENTS}

This work has received funding from the European Union's Horizon 2020 research and innovation program under grant agreement No 965417.

\section{REFERENCES}

1. Loscalzo J, Barabasi AL. Systems biology and the future of medicine. Wiley Interdiscip Rev Syst Biol Med 2011;3(6):619-27.

2. Vanfleteren LE, Kocks JW, Stone IS, et al. Moving from the Oslerian paradigm to the post-genomic era: are asthma and COPD outdated terms? Thorax 2014;69(1):72-9.

3. Shepard JW Jr, Buysse DJ, Chesson AL Jr, et al, History of the development of sleep medicine in the United States. J Clin Sleep Med 2005;1(1): 61-82.
4. Penzel T, Pevernagie D, Bassetti C, et al. Sleep medicine catalogue of knowledge and skills - Revision. J Sleep Res 2021;30(3):e13394.

5. Mayer G, Pevernagie D. Nosological classification and diagnostic strategy. In: Overeem S, Reading P, editors. Sleep disorders in neurology. A practical approach. 2 ed. Chichester, UK: Wiley-Blackwell; 2018. p. 41-52.

6. Pevernagie DA, Gnidovec-Strazisar B, Grote L, et al. On the rise and fall of the apnea-hypopnea index: a historical review and critical appraisal. J Sleep Res 2020;29(4):e13066.

7. ASDA. Obstructive sleep apnea syndrome (780.53-0). In: The international classification of sleep disorders diagnostic and coding manual. 1 ed. Rochester, MN, USA: American Sleep Disorders Association; 1990. p. 52-8.

8. AASM. Obstructive sleep apnea, adult. In: Sateia M, Hauri $P$, editors. The international classification of sleep disorders - diagnostic and coding manual. 2 ed. Westchester, IL, USA: American Academy of Sleep Medicine; 2005. p. 51-5.

9. AASM. Sleep-related breathing disorders in adults: recommendations for syndrome definition and measurement techniques in clinical research. The Report of an American Academy of Sleep Medicine Task Force. Sleep 1999;22(5):667-89.

10. Young T, Peppard P, Palta M, et al. Population-based study of sleep-disordered breathing as a risk factor for hypertension. Arch Intern Med 1997;157(15):1746-52.

11. Damer TE. Attacking faulty reasoning. 6 ed. Belmont, USA: Wadsworth; 2009.

12. Heller J. Catch-22. London, UK: Vintage; 1994.

13. Heinzer R, Vat S, Marques-Vidal P, et al. Prevalence of sleep-disordered breathing in the general population: the HypnoLaus study. Lancet Respir Med 2015; 3(4):310-8.

14. O'Mahony S. Medicine and the McNamara fallacy. J R Coll Physicians Edinb 2017;47(3):281-7.

15. AASM. Obstructive sleep apnea, adult. In: Sateia M, editor. The international classification of sleep disorders. 3 ed. Darien, IL, USA: American Academy of Sleep Medicine; 2014. p. 53-62.

16. Kapur VK, Auckley DH, Chowdhuri S, et al. Clinical practice guideline for diagnostic testing for adult obstructive sleep apnea: an American Academy of sleep medicine clinical practice guideline. J Clin Sleep Med 2017;13(3):479-504.

17. Randerath W, Bassetti CL, Bonsignore MR, et al. Challenges and perspectives in obstructive sleep apnoea: Report by an ad hoc working group of the sleep disordered breathing group of the European respiratory Society and the European sleep research Society. Eur Respir J 2018;52(3):1702616.

18. Ahn AC, Tewari M, Poon CS, et al. The limits of reductionism in medicine: could systems biology offer an alternative? Plos Med 2006;3(6):e208. 
19. Agusti A, Bel E, Thomas M, et al. Treatable traits: toward precision medicine of chronic airway diseases. Eur Respir J 2016;47(2):410-9.

20. Agusti A. Phenotypes and disease characterization in chronic obstructive pulmonary disease. Toward the extinction of phenotypes? Ann Am Thorac Soc 2013;10(Suppl):S125-30.

21. Agusti A. The path to personalised medicine in COPD. Thorax 2014;69(9):857-64.

22. Jameson JL, Longo DL. Precision medicine-personalized, problematic, and promising. N Engl J Med 2015;372(23):2229-34.

23. Ahn AC, Tewari M, Poon CS, et al. The clinical applications of a systems approach. Plos Med 2006;3(7): e209.

24. Agusti A, Celli B, Faner R. What does endotyping mean for treatment in chronic obstructive pulmonary disease? Lancet 2017;390(10098):980-7.

25. Pack Al. Application of personalized, predictive, Preventative, and Participatory (P4) medicine to obstructive sleep apnea. A Roadmap for improving Care? Ann Am Thorac Soc 2016;13(9):1456-67.

26. Martinez-Garcia MA, Campos-Rodriguez F, Barbe F, et al. Precision medicine in obstructive sleep apnoea. Lancet Respir Med 2019;7(5):456-64.

27. Edwards BA, Redline S, Sands SA, et al. More than the Sum of the respiratory events: personalized medicine approaches for obstructive sleep apnea. Am J Respir Crit Care Med 2019;200(6):691-703.

28. Eckert DJ, White DP, Jordan AS, et al. Defining phenotypic causes of obstructive sleep apnea. Identification of novel therapeutic targets. Am J Respir Crit Care Med 2013;188(8):996-1004.

29. Carberry JC, Amatoury J, Eckert DJ. Personalized management approach for OSA. Chest 2018; 153(3):744-55.

30. Eckert DJ. Phenotypic approaches to obstructive sleep apnoea - new pathways for targeted therapy. Sleep Med Rev 2018;37:45-59.

31. Owens RL, Edwards BA, Eckert DJ, et al. An integrative model of physiological traits can be used to predict obstructive sleep apnea and response to non positive airway pressure therapy. Sleep 2015;38(6): 961-70.

32. Han MK, Agusti A, Calverley PM, et al. Chronic obstructive pulmonary disease phenotypes: the future of COPD. Am J Respir Crit Care Med 2010; 182(5):598-604.

33. Zinchuk AV, Gentry MJ, Concato J, et al. Phenotypes in obstructive sleep apnea: a definition, examples and evolution of approaches. Sleep Med Rev 2017;35:113-23.
34. Zinchuk A, Yaggi HK. Phenotypic subtypes of OSA: a challenge and opportunity for precision medicine. Chest 2020;157(2):403-20.

35. Ye L, Pien GW, Ratcliffe SJ, et al. The different clinical faces of obstructive sleep apnoea: a cluster analysis. Eur Respir J 2014;44(6):1600-7.

36. Keenan BT, Kim J, Singh B, et al. Recognizable clinical subtypes of obstructive sleep apnea across international sleep centers: a cluster analysis. Sleep 2018;41(3):zsx214.

37. Saaresranta T, Hedner J, Bonsignore MR, et al. Clinical phenotypes and comorbidity in European sleep apnoea patients. PLoS One 2016;11(10):e0163439.

38. Mazzotti DR, Keenan BT, Lim DC, et al. Symptom subtypes of obstructive sleep apnea predict incidence of cardiovascular outcomes. Am J Respir Crit Care Med 2019;200(4):493-506.

39. Zinchuk A, Yaggi HK. Sleep apnea heterogeneity, phenotypes, and cardiovascular risk. Implications for trial Design and precision sleep medicine. Am J Respir Crit Care Med 2019;200(4):412-3.

40. Sands SA, Edwards BA, Terrill PI, et al. Phenotyping Pharyngeal pathophysiology using polysomnography in patients with obstructive sleep apnea. Am J Respir Crit Care Med 2018;197(9):1187-97.

41. Bosi M, De Vito A, Kotecha B, et al. Phenotyping the pathophysiology of obstructive sleep apnea using polygraphy/polysomnography: a review of the literature. Sleep Breath 2018;22(3):579-92.

42. Lim DC, Mazzotti DR, Sutherland K, et al. Reinventing polysomnography in the age of precision medicine. Sleep Med Rev 2020;52:101313.

43. Stephansen JB, Olesen AN, Olsen M, et al. Neural network analysis of sleep stages enables efficient diagnosis of narcolepsy. Nat Commun 2018;9(1): 5229.

44. Sanchez-de-la-Torre M, Khalyfa A, Sanchez-de-laTorre A, et al. Precision medicine in patients with resistant hypertension and obstructive sleep apnea: blood pressure response to continuous positive airway pressure treatment. J Am Coll Cardiol 2015; 66(9): 1023-32.

45. Pinilla L, Barbe F, de Gonzalo-Calvo D. MicroRNAs to guide medical decision-making in obstructive sleep apnea: a review. Sleep Med Rev 2021;59: 101458.

46. De Luca Canto G, Pacheco-Pereira C, Aydinoz S, et al. Biomarkers associated with obstructive sleep apnea and morbidities: a scoping review. Sleep Med 2015;16(3):347-57. 